\title{
Immunohistochemical investigation of tumorigenic pathways in small intestinal adenocarcinoma: a comparison with colorectal adenocarcinoma
}

\author{
Megan Q Zhang, Zong-Ming E Chen and Hanlin L Wang \\ Lauren V Ackerman Laboratory of Surgical Pathology, Department of Pathology and Immunology, \\ Washington University School of Medicine, St Louis, MO, USA
}

\begin{abstract}
Small intestinal adenocarcinoma is an uncommon neoplasm morphologically similar to or indistinguishable from colorectal adenocarcinoma. Although much has been learned about genetic pathways critical to colorectal tumorigenesis, little is known about molecular alterations involved in the development of small intestinal adenocarcinoma. In this study, we immunohistochemically compared non-ampullary small intestinal adenocarcinomas with sporadic colorectal adenocarcinomas for the expression of several proteins known to serve pivotal roles in colorectal tumorigenesis. These included adenomatous polyposis coli and $\beta$-catenin involved in the Wnt signaling pathway, and DNA mismatch repair enzymes hMLH1, hMSH2 and hMSH6 involved in the microsatellite instability pathway. The expression of two important tumor suppressors, p53 and RB, was also examined. The results show that complete loss of adenomatous polyposis coli immunoreactivity, presumably resulting from its gene mutations, was observed in eight of $26(31 \%)$ small intestinal adenocarcinomas and 36 of $51(71 \%)$ colorectal adenocarcinomas $(P=0.0008)$. Nuclear localization of $\beta$-catenin, an indirect evidence of deregulated Wnt signaling pathway, was observed in $5(19 \%)$ small intestinal adenocarcinomas and $36(71 \%)$ colorectal adenocarcinomas $(P<\mathbf{0 . 0 0 0 1})$. Total lack of nuclear staining for one or more of the DNA mismatch repair enzymes occurred in a similar low frequency in both small intestinal and colorectal adenocarcinomas, seen in two of $25(8 \%)$ and 10 of $47(21 \%)$ cases, respectively $(P=0.1958)$. The frequencies of aberrant p53 and RB expression were also similar between small intestinal and colorectal adenocarcinomas. These observations indicate that defects in the Wnt and microsatellite instability pathways occur in over $90 \%$ of colorectal adenocarcinomas, but in only $40 \%$ of small intestinal adenocarcinomas. Small intestinal tumorigenesis appears to follow a distinct, yet unidentified, molecular pathway(s) from its colorectal counterpart despite their morphologic similarity.
\end{abstract}

Modern Pathology (2006) 19, 573-580. doi:10.1038/modpathol.3800566; published online 24 February 2006

Keywords: small intestine; colorectal; adenocarcinoma; Wnt; microsatellite instability

Primary adenocarcinoma of the small intestine is morphologically similar to or indistinguishable from colorectal adenocarcinoma. It also shares many of the risk factors with colorectal adenocarcinoma in tumorigenesis, such as familial adenomatous polyposis, hereditary nonpolyposis colorectal cancer syndrome, Peutz-Jeghers syndrome and Crohn's

Correspondence: Dr HL Wang, MD, PhD, Lauren V Ackerman Laboratory of Surgical Pathology, Department of Pathology and Immunology, Washington University School of Medicine, Campus Box 8118, 660 South Euclid Avenue, St Louis, MO 63110-1093, USA.

E-mail: hwang@path.wustl.edu

Received 15 November 2005; revised and accepted 13 January 2006; published online 24 February 2006 disease. ${ }^{1}$ However, small intestinal adenocarcinoma occurs 50 times less frequently than colorectal adenocarcinoma, even though the small intestine makes up approximately $75 \%$ of the length and $90 \%$ of the mucosal surface of the gastrointestinal tract. ${ }^{2}$ A clear explanation for this puzzling disparity has not been well established, although several suggestions have been proposed. One possibility is the rapid transit and diluted nature of intraluminal contents in the small intestine, which reduces the exposure time of enterocytes to carcinogens. Low bacterial counts in the small intestine may also minimize the formation of carcinogenic substances from bacterial degradation of bile components. Furthermore, rapid turnover of small intestinal epithelial cells may competitively inhibit the 
overgrowth of neoplastically transformed cells, and local immune response unique to the small intestine may also contribute to the suppression of tumor development. ${ }^{3}$

Despite the rapid advance in the understanding of molecular pathways involved in the development of colorectal adenocarcinoma, little is known about small intestinal tumorigenesis. Given the morphologic similarity and common risk factors, one would presume that small intestinal and colorectal adenocarcinomas might share the same or similar genetic and molecular alternations. However, we have recently demonstrated that small intestinal adenocarcinoma differs markedly from colorectal counterpart immunophenotypically. In contrast to the classic cytokeratin (CK) 7-negative/ CK20-positive colorectal pattern, a significant number of small intestinal adenocarcinomas lose CK20 immunoreactivity while aberrantly expressing CK7. ${ }^{4}$ The alterations in CK7 and CK20 expression occur early in small intestinal tumorigenesis and are observed in adenomatous epithelium. These observations are intriguing because the normal small intestinal mucosa shows the same CK7negative/CK20-positive profile as its colorectal counterpart. Small intestinal adenocarcinoma also differs from colorectal adenocarcinoma in the expression of $\alpha$-methylacyl coenzyme A racemase, which is frequently overexpressed in colorectal adenocarcinomas, but only rarely detected in small intestinal adenocarcinomas. ${ }^{5}$ Taken together, these observations suggest that small intestinal tumorigenesis involves distinct molecular mechanisms.

To further understand the small intestinal tumorigenesis, the current study was undertaken to examine the immunohistochemical evidence of deregulation of molecular pathways that have been well characterized in colorectal tumorigenesis. These included adenomatous polyposis coli and $\beta$-catenin proteins that serve key roles in the $\mathrm{Wnt}$ signaling pathway, and DNA mismatch repair enzymes hMLH1, hMSH2 and hMSH6 involved in the microsatellite instability pathway. The expression of two other important tumor suppressors, p53 and RB, was also examined.

\section{Materials and methods}

\section{Case Selection}

Twenty-six cases of surgically resected primary adenocarcinoma of the small intestine were collected from the pathology archives of Washington University Barnes-Jewish Hospital spanning the years 1989-2003. These included two cases from the duodenum, 17 from the jejunum, and seven from the ileum. Clinical data were reviewed to ensure that they were indeed small intestinal primaries. Cases with any known history of adenocarcinoma in other organs were excluded from this study. Tumors arising from the ampullary region were also excluded because of their potential pancreaticobiliary origin. For comparison, 51 cases of surgically resected sporadic colorectal adenocarcinomas were included in this study. These included 23 tumors secondarily involving the small intestine by either direct extension or metastasis. The remaining 28 cases were primary colorectal adenocarcinomas randomly selected from the 2000-2002 surgical pathology archives from patients who did not receive preoperative neoadjuvant chemoradiation therapy and did not have a known history of familial adenomatous polyposis, hereditary nonpolyposis colorectal cancer syndrome or inflammatory bowel disease. Hematoxylin-eosin (H\&E)-stained slides were reviewed to confirm the diagnosis. Formalinfixed, paraffin-embedded tissue blocks were selected to include adenocarcinoma and normalappearing non-neoplastic intestinal mucosa.

\section{Immunohistochemistry}

Immunohistochemical studies were performed on $4-\mu \mathrm{m}$ tissue sections using a panel of commercially available antibodies listed in Table 1. Immunostaining was conducted employing the LSAB Plus system purchased from DAKO Corp (Carpinteria, CA, USA) and the ABC kit purchased from Vector Laboratories (Burlingame, CA, USA), following the manufacturers' instructions with slight modifications. Briefly, deparaffinized tissue sections were first treated with $3 \% \mathrm{H}_{2} \mathrm{O}_{2}$ for $15 \mathrm{~min}$ to inhibit endo-

Table 1 Antibodies used for immunohistochemical analysis

\begin{tabular}{llcc}
\hline Specificity & Source (clone) & Dilution & Antigen retrieval \\
\hline APC, carboxyl terminus & Santa Cruz (C-20, polyclonal) & $1: 800$ & Citrate, MW 14 min \\
$\beta$-Catenin & Santa Cruz (15) & $1: 100$ & Citrate, MW 10 min \\
hMLH1 & PharMingen (G168-728) & $1: 20$ & Citrate, MW 15 min \\
hMSH2 & Zymed (FE11) & $1: 100$ & EDTA, MW 10 min \\
hMSH6 & Biocare Medical (BC/44) & $1: 10$ & Citrate, MW 15 min \\
p53 & Santa Cruz (DO-1) & $1: 100$ & Citrate, MW 8 min \\
RB & Santa Cruz (IF8) & $1: 100$ & Citrate, MW 25 min
\end{tabular}

Santa Cruz Biotechnology, Santa Cruz, CA, USA; PharMingen, San Diego, CA, USA; Zymed Laboratories, Inc., South San Francisco, CA, USA; Biocare Medical, Walnut Creek, CA, USA; Citrate buffer, $10 \mathrm{mmol} / \mathrm{l}$, pH 6.0; EDTA solution, $1 \mathrm{mmol} / 1$, pH 8.0 .

APC, adenomatous polyposis coli; MW, microwave heating; EDTA, ethylenediaminetetraacetic acid. 
genous peroxidase followed by antigen retrieval under variable conditions specified in Table 1. After incubation with blocking serum for $20 \mathrm{~min}$, sections were incubated with the primary antibody for $1 \mathrm{~h}$ at room temperature, followed by further incubation with biotinylated link antibody and peroxidaselabeled streptavidin. The staining was developed by reaction with 3,3'-diaminobenzidine substratechromogen solution followed by counterstaining with hematoxylin 7211 purchased from RichardAllan Scientific (Kalamazoo, MI, USA). In each experiment, a negative control was included in which the primary antibody was replaced by non-human-reactive mouse or rabbit IgG. Positive controls used in this study were sections from a colorectal adenocarcinoma that also contained adjacent non-neoplastic colonic mucosa.

The immunostaining patterns (membranous, cytoplasmic or nuclear) were independently analyzed by two observers (MQZ and HLW, or Z-MEC and HLW). Cases with discrepant interpretation were resolved by re-review by all three investigators. In general, a tumor was considered negative if less than $5 \%$ of the tumor cells exhibited immunoreactivity. For some of the immunomarkers, as specified in the Results section, positive staining was further stratified as diffuse if more than $50 \%$ of the tumor cells showed immunoreactivity, and focal if $5-50 \%$ of the tumor cell stained.

\section{Statistical Analysis}

Statistical analysis was performed using the Statistica software for windows (StatSoft, Inc., Tulsa, OK, USA). A $P$-value of $<0.05$, as determined by twotailed Fisher exact test or the $\chi^{2}$ test with Yates continuity correction, was considered statistically significant.

\section{Results}

Expression of Adenomatous Polyposis Coli and $\beta$-Catenin Proteins in Small Intestinal and Colorectal Adenocarcinomas

Positive membranous and cytoplasmic staining was universally detected in normal-appearing non-neoplastic small intestinal and colorectal epithelial cells using a rabbit polyclonal antibody (C-20) raised against the carboxyl terminus of the adenomatous polyposis coli protein. Complete absence of immunoreactivity in tumor cells in the presence of positive staining in normal mucosa in the same sections (Figure 1), presumably resulting from mutations of the adenomatous polyposis coli gene, was observed in eight of 26 small intestinal adenocarcinomas $(31 \%)$ and 36 of 51 colorectal adenocarcinomas $(71 \%)$, respectively $(P=0.0008)$.

Nuclear staining for $\beta$-catenin, an indirect evidence of deregulation of the Wnt signaling pathway

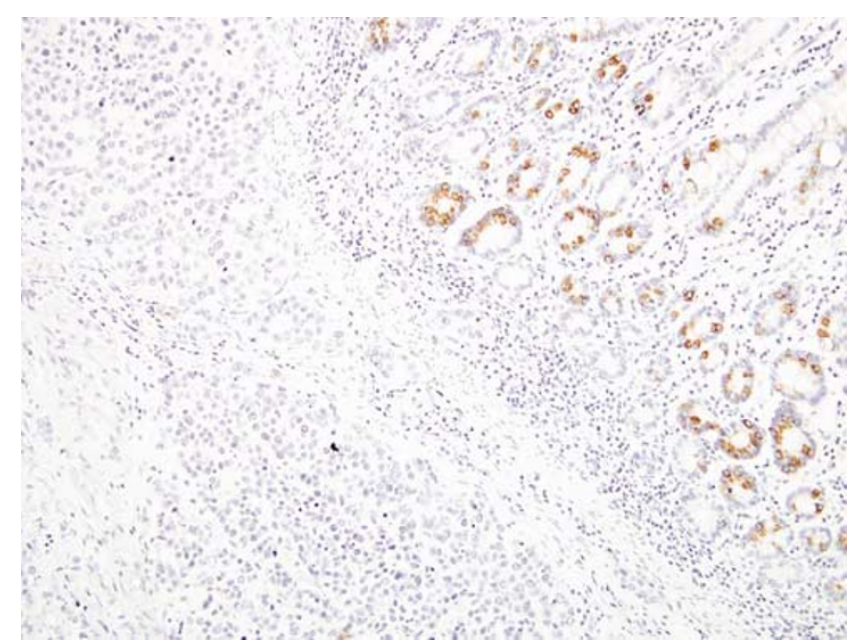

Figure 1 Complete lack of immunostaining for adenomatous polyposis coli seen in a small intestinal adenocarcinoma (left lower) with positive membranous and cytoplasmic staining in normal-appearing small intestinal mucosa (right upper) (original magnification $\times 200$ ).

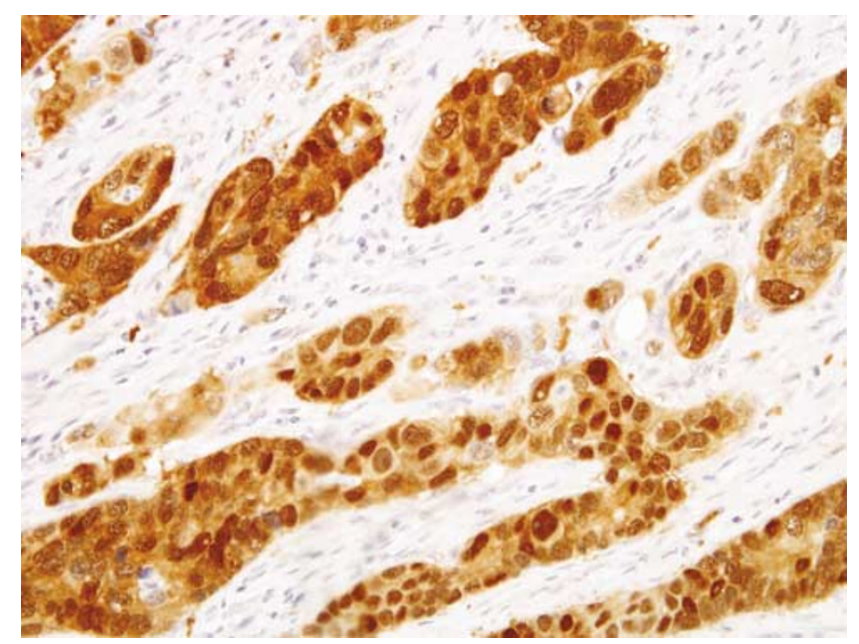

Figure 2 Strong nuclear staining for $\beta$-catenin seen in a small intestinal adenocarcinoma. Note that strong cytoplasmic staining was also present (original magnification $\times 400$ ).

usually secondary to adenomatous polyposis coli or $\beta$-catenin mutations, was detected in five small intestinal adenocarcinomas $(19 \%)$ and 36 colorectal adenocarcinomas $(71 \%)$, respectively $(P<0.0001)$. These tumors also typically exhibited a strong cytoplasmic immunoreactivity for $\beta$-catenin (Figure 2). This was in marked contrast to non-neoplastic small intestinal and colorectal epithelial cells where only membranous staining (usually with a weaker staining intensity) was evident (Figure 3). Nuclear staining was never observed in normal-appearing intestinal mucosa. In colorectal adenocarcinomas, diffuse nuclear $\beta$-catenin staining was seen in 21 cases $(58 \%)$, in contrast to only one small intestinal adenocarcinoma, although this difference was not statistically significant $(P=0.1644)$. In the rest of the 
tumors that showed $\beta$-catenin nuclear localization, a focal staining pattern was observed.

Table 2 summarizes the immunohistochemical findings of adenomatous polyposis coli and $\beta$-catenin expression in small intestinal and colorectal adenocarcinomas. A relatively higher frequency of concurrent loss of adenomatous polyposis coli immunoreactivity and nuclear expression

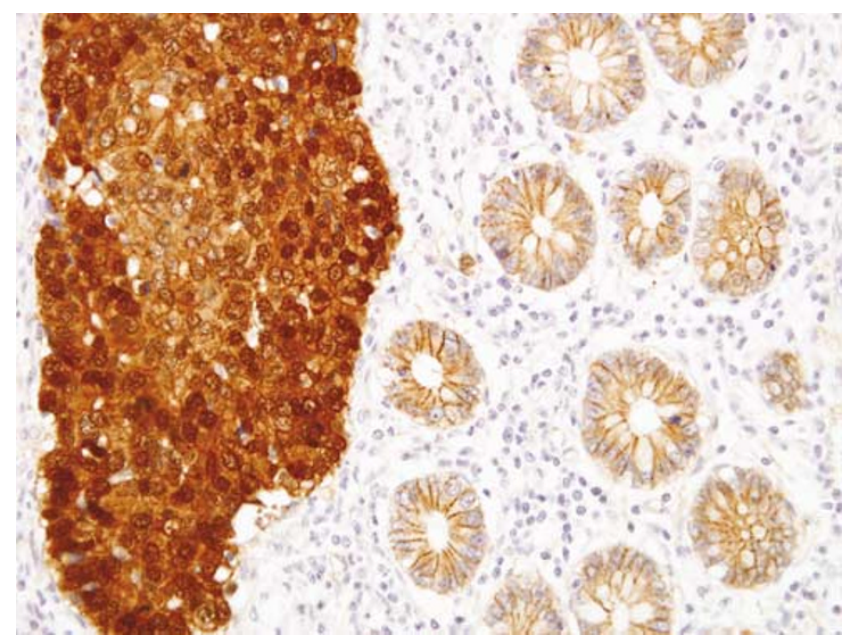

Figure 3 Strong nuclear and cytoplasmic staining of $\beta$-catenin seen in a colorectal adenocarcinoma (left). Note membranous staining in normal-appearing colonic mucosa (right). No nuclear staining was observed in non-neoplastic intestinal epithelium (original magnification $\times 400$ ). of $\beta$-catenin was seen in colorectal adenocarcinomas, noted in 31 cases $(86 \%)$ relative to four small intestinal adenocarcinomas $(50 \% ; P=0.0422)$. It is interesting to note that occurrence of nuclear $\beta$-catenin expression without loss of adenomatous polyposis coli immunoreactivity was observed in only one small intestinal adenocarcinoma (4\%) and five colorectal adenocarcinomas (10\%). Thus, the total number of cases showing immunohistochemical evidence of deregulation of the Wnt signaling pathway, as defined by either negative adenomatous polyposis coli staining, $\beta$-catenin nuclear localization, or both, was $9(35 \%)$ and $41(80 \%)$ for small intestinal adenocarcinoma and colorectal adenocarcinoma, respectively $(P=0.0001)$.

\section{Expression of DNA Mismatch Repair Enzymes in Small Intestinal and Colorectal Adenocarcinomas}

As shown in Table 3, total lack of nuclear staining for one or more of the three DNA mismatch repair enzymes in tumor cells in the presence of positive immunoreactivity in non-neoplastic cells (eg, normal-appearing epithelial cells, stromal cells, or inflammatory cells), defined as microsatellite instable, was demonstrated in two of 25 small intestinal adenocarcinomas $(8 \%)$ and 10 of 47 colorectal adenocarcinomas $(21 \%)$ with interpretable staining $(P=0.1958)$. The remaining tumors were microsatellite stable as evidenced by positive

Table 2 Summary of immunohistochemical findings of adenomatous polyposis coli (APC) and $\beta$-catenin expression in small intestinal and colorectal adenocarcinomas

\begin{tabular}{|c|c|c|c|c|c|}
\hline \multirow[t]{3}{*}{ Immunoreactivity } & \multicolumn{5}{|c|}{ No. (\%) of cases } \\
\hline & \multicolumn{3}{|c|}{ Small intestinal } & \multicolumn{2}{|c|}{ Colorectal } \\
\hline & Duodenum $(\mathrm{n}=2)$ & Jejunum $(\mathrm{n}=17)$ & Ileum $(\mathrm{n}=7)$ & Primary $(\mathrm{n}=28)$ & Secondary $(\mathrm{n}=23)$ \\
\hline APC, negative staining & $1(50)$ & $5(29)$ & $2(29)$ & $21(75)$ & $15(65)$ \\
\hline$\beta$-Catenin, nuclear staining & $1(50)$ & $3(18)$ & $1(14)$ & $22(79)$ & $14(61)$ \\
\hline Both $^{\mathrm{a}}$ & $1(50)$ & $2(12)$ & $1(14)$ & $20(71)$ & $11(48)$ \\
\hline
\end{tabular}

${ }^{\mathrm{a}}$ Concurrent loss of adenomatous polyposis coli immunoreactivity and gain of nuclear $\beta$-catenin expression in the same tumor.

Table 3 Summary of immunohistochemical findings of expression of DNA mismatch repair enzymes in small intestinal and colorectal adenocarcinomas

\begin{tabular}{|c|c|c|c|c|c|}
\hline \multirow[t]{3}{*}{ Immunoreactivity } & \multicolumn{5}{|c|}{ No. (\%) of cases } \\
\hline & \multicolumn{3}{|c|}{ Small intestinal } & \multicolumn{2}{|c|}{ Colorectal } \\
\hline & Duodenum $(\mathrm{n}=2)$ & Jejunum $(\mathrm{n}=16)$ & Ileum $(\mathrm{n}=7)$ & Primary $(\mathrm{n}=24)$ & Secondary $(\mathrm{n}=23)$ \\
\hline hMLH1, negative staining & 0 & $1(6)$ & $1(14)$ & $3(13)$ & $6(26)$ \\
\hline hMSH2, negative staining & 0 & 0 & 0 & 0 & $1(4)^{\mathrm{a}}$ \\
\hline hMSH6, negative staining & 0 & 0 & 0 & 0 & $1(4)$ \\
\hline
\end{tabular}

${ }^{\mathrm{a}}$ This tumor also lacked immunostaining for hMLH1. 
nuclear staining for all three enzymes (Figure 4). Both microsatellite instable small intestinal adenocarcinomas lacked immunohistochemical expression for hMLH1. Among those 10 microsatellite instable colorectal adenocarcinomas, eight tumors lacked staining for hMLH1 (Figure 5), one for hMLH1 and hMSH2 concurrently, and one for hMSH6. Alhough statistically insignificant $(P=0.1332)$, secondary colorectal adenocarcinomas appeared to show a higher frequency of microsatellite instability when compared with primary colorectal adenocarcinomas (Table 3), presumably due to the fact that the vast majority of the secondary

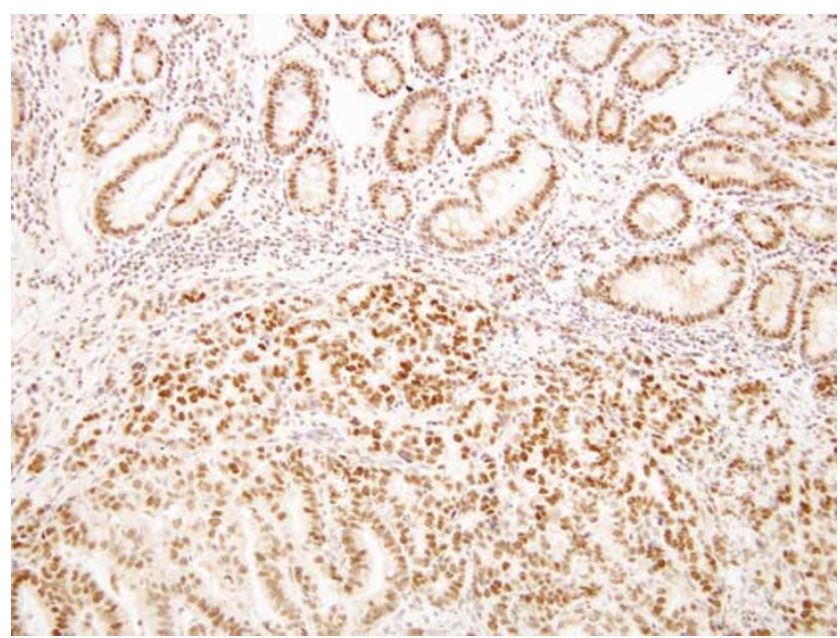

Figure 4 An example of microsatellite stable tumor (small intestinal adenocarcinoma) showing diffuse nuclear staining for hMLH2 in both adenocarcinomatous component (lower), normalappearing small intestinal epithelium (upper) and inflammatory cells (original magnification $\times 200$ ).

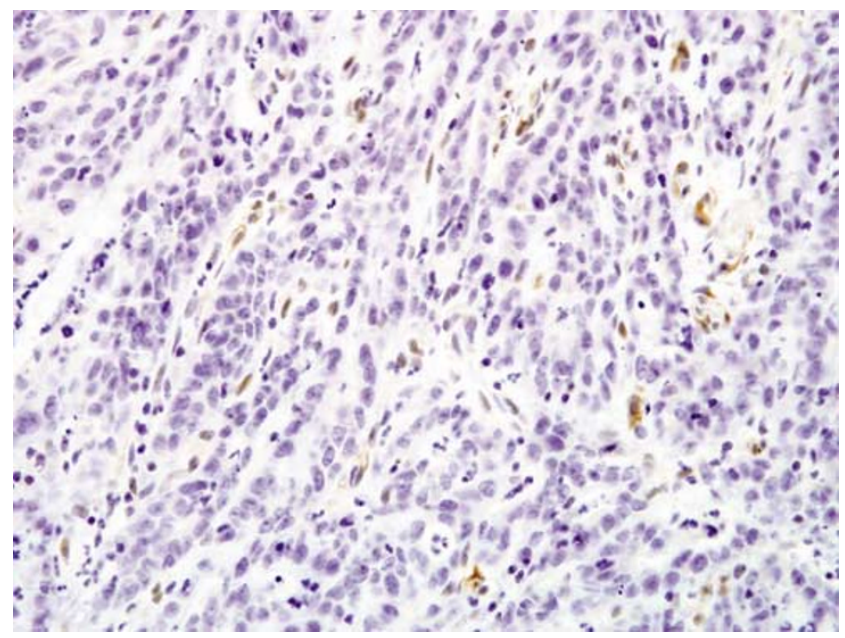

Figure 5 An example of microsatellite instable tumor (colorectal adenocarcinoma) showing negative staining for hMLH1 in adenocarcinomatous component, while intermixed non-neoplastic cells (stromal cells, inflammatory cells and endothelial cells) showed positive nuclear staining to serve as built-in controls (original magnification $\times 400$ ). tumors were right-sided and involved the small intestine by direct extension, whereas primary colorectal adenocarcinomas examined in this study were randomly selected from various portions of the colon.

The microsatellite instability status and loss of adenomatous polyposis coli immunoreactivity were essentially mutually exclusive in both small intestinal and colorectal adenocarcinomas. In only two colorectal adenocarcinomas was loss of staining for both adenomatous polyposis coli and hMLH1 proteins observed.

\section{Expression of p53 and RB Proteins in Small Intestinal and Colorectal Adenocarcinomas}

Strong and diffuse nuclear staining for p53 in tumor cells in the complete absence of nuclear expression in normal-appearing non-neoplastic intestinal mucosa (Figure 6) was detected in 14 of 26 small intestinal adenocarcinomas (54\%) and 10 of 23 colorectal adenocarcinomas (44\%), respectively $(P=0.4687)$. In the remaining cases, the staining was either focally positive or completely negative. Although no correlation was observed between p53 overexpression and aberrant adenomatous polyposis coli and/or $\beta$-catenin immunostaining, microsatellite instable tumors tended to show either negative or only focal nuclear staining for p53. In nine microsatellite instable tumors (two small intestinal and seven colorectal adenocarcinomas) examined for p53 expression, only one colorectal adenocarcinoma exhibited a strong and diffuse staining pattern $(P=0.0232)$.

Loss of nuclear RB protein expression in tumor cells in the presence of nuclear staining in nonneoplastic cells (Figure 7) was an infrequent event in both types of intestinal neoplasms. In 24 small

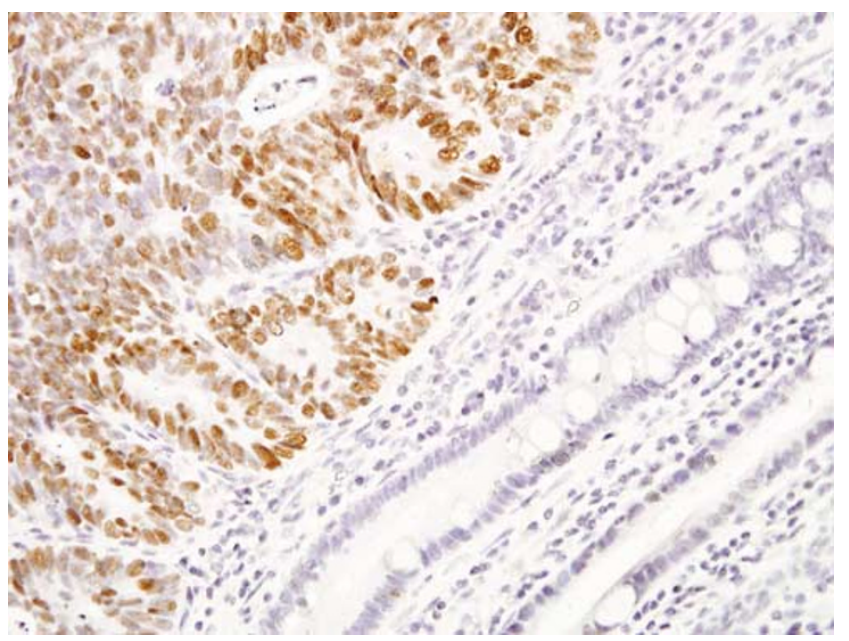

Figure 6 Strong and diffuse nuclear staining for p53 seen in a small intestinal adenocarcinoma (left upper), with complete negative staining in normal-appearing small intestinal mucosa (right lower) (original magnification $\times 400$ ). 
578

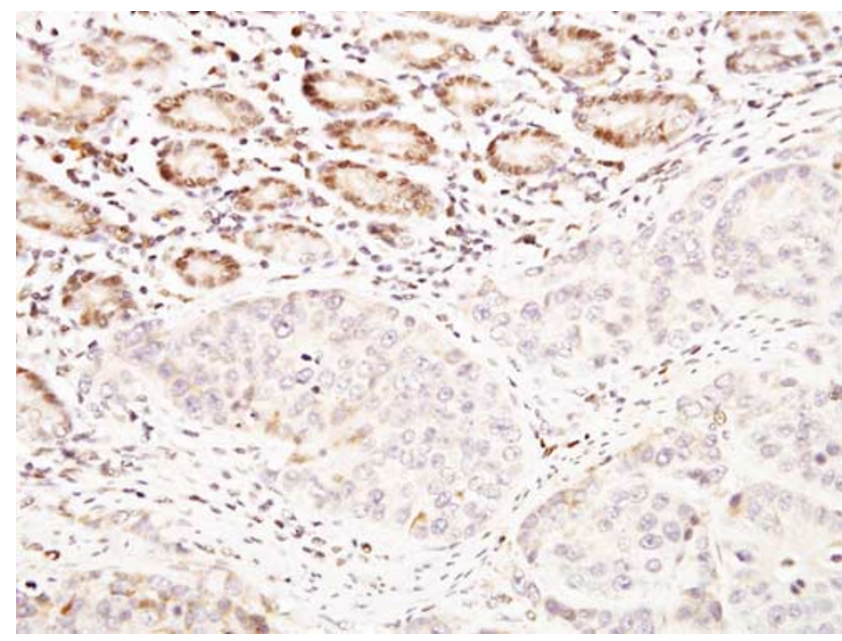

Figure 7 Loss of nuclear RB staining seen in a small intestinal adenocarcinoma (lower right), in the presence of positive nuclear staining in normal-appearing small intestinal mucosa (upper left). Note that only weak cytoplasmic immunoreactivity was observed in tumor cells (original magnification $\times 400$ ).

intestinal adenocarcinomas and 23 colorectal adenocarcinomas examined in this study, loss of RB nuclear staining was seen in only $2(8 \%)$ and 1 $(4 \%)$ cases, respectively $(P=0.5763)$.

\section{Discussion}

Deregulation of the Wnt signaling pathway as a key event in the initiation phase of human colorectal tumorigenesis has been well established by numerous studies. ${ }^{6,7}$ This primarily involves mutations of the adenomatous polyposis coli tumor suppressor gene, which occur in $70-80 \%$ of colorectal epithelial neoplasms. A critical function of the wild-type adenomatous polyposis coli protein is to facilitate phosphorylation and subsequent degradation of $\beta$-catenin, a membranous protein involved in cell adhesion. $^{7,8}$ Loss of adenomatous polyposis coli function by mutations or deletion, as seen in the majority of colorectal adenocarcinomas, results in $\beta$-catenin stabilization, cytoplasmic accumulation and nuclear translocation. In the nucleus, $\beta$-catenin acts as a transactivating factor for the transcription factor Tcf-4 to regulate the transcription of a number of downstream target genes that are believed to be the mediators of neoplastic transformation. ${ }^{7}$ In a small subset of colorectal adenocarcinomas that possess a wild-type adenomatous polyposis coli gene, a similar transactivation mechanism occurs directly via $\beta$-catenin mutation that also prevents its protein degradation. ${ }^{9}$ Therefore, $\beta$-catenin is a dual function protein and its nuclear localization is a crucial step in activation of the Wnt signaling pathway that leads to tumorigenesis.

Another well-established molecular alteration in the development of colorectal adenocarcinoma is microsatellite instability, characterized by accumu- lation of changes in the length of simple repeated nucleotide sequences known as microsatellites secondary to mutations of DNA mismatch repair genes such as those encoding mismatch repair enzymes hMLH1, hMSH2 and hMSH6. Although microsatellite instability is a hallmark of hereditary nonpolyposis colorectal cancer syndrome where mutations of one or more DNA mismatch repair genes are found in greater than $90 \%$ of the cases, it is detected in $10-15 \%$ of sporadic colorectal adenocarcinomas. ${ }^{10-12}$

To investigate whether small intestinal adenocarcinoma shares the same molecular alterations with colorectal adenocarcinoma, we took advantage of immunohistochemical staining that has proven to be useful tools to indirectly detect gene mutations. For example, the antibody used to detect adenomatous polyposis coli protein only recognizes its carboxyl terminus and the vast majority of mutations in the adenomatous polyposis coli gene have been shown to result in truncated proteins with a lack of the carboxyl terminus. Thus, lack of immunoreactivity in tumor cells in the presence of positive staining in non-neoplastic cells would indicate the presence of gene mutations. ${ }^{13,14}$

Our study demonstrates a significant lower frequency of negative immunostaining for adenomatous polyposis coli in small intestinal adenocarcinoma than in colorectal adenocarcinoma (31 vs $71 \%$ ). However, this frequency appears to be somewhat higher than those reported by others using genetic or molecular approaches. In the study by Rashid and Hamilton, ${ }^{15}$ allelic loss at chromosome $5 \mathrm{q}$, the region of the adenomatous polyposis coli gene, was found to be extremely rare, detected in only one of 21 small intestinal adenocarcinomas $(5 \%)$. Arai et $a l^{16}$ and Bläker et $a l^{17}$ demonstrated mutations in the adenomatous polyposis coli gene in 3 of a total of 36 small intestinal adenocarcinomas $(8 \%)$ they examined. Furthermore, Wheeler et al ${ }^{18}$ did not find any mutation in 21 cases. It should be pointed out, however, that detection of mutations in the adenomatous polyposis coli gene was usually performed by polymerase chain reaction in these studies and only the mutation cluster region was screened. Although a large proportion of the truncating mutations in colorectal adenocarcinoma occur within the mutation cluster region, many mutations are widespread throughout this very large gene. ${ }^{19,20}$ It is therefore highly likely that in small intestinal adenocarcinoma some of the mutations are also present outside the mutation cluster region, which were not detected in these studies. Apparently, it is not a sensitive method to analyze adenomatous polyposis coli mutations by testing allelic loss at $5 \mathrm{q}^{21}$ In this regard, immunohistochemical staining may be more sensitive because it stains adenomatous polyposis coli protein only when its carboxyl terminus is preserved. Theoretically, any mutation that results in truncation or absence of the protein would lead to negative 
staining. This presumption is confirmed by our findings that $71 \%$ colorectal adenocarcinomas show negative immunostaining for adenomatous polyposis coli, in keeping with current knowledge of the frequency of gene mutations in sporadic colorectal adenocarcinomas. ${ }^{7,22}$

Similar to that seen for adenomatous polyposis coli, the frequency of nuclear $\beta$-catenin expression is also much lower in small intestinal adenocarcinoma when compared with colorectal adenocarcinoma. Nevertheless, nuclear staining is observed only in tumor cells and never seen in non-neoplastic epithelial cells. This is true of all small intestinal and colorectal adenocarcinomas that exhibit aberrant $\beta$-catenin expression. It should be stressed that in both small intestinal and colorectal adenocarcinomas, tumors with nuclear $\beta$-catenin expression show a very high frequency of concordant loss of adenomatous polyposis coli immunoreactivity, seen in 80 and $86 \%$ of the cases, respectively. These observations indicate that, as with colorectal adenocarcinoma, nuclear translocation of $\beta$-catenin protein detected in small intestinal adenocarcinoma also mainly results from adenomatous polyposis coli mutations. Our findings are also in accord with those reported by other investigators who examined $\beta$-catenin expression in small intestinal adenocarcinoma by immunohistochemistry. In the studies by Bläker et $a l,{ }^{17}$ Wheeler et $a l,{ }^{18}$ and Svrcek et $a l,{ }^{23}$ nuclear $\beta$-catenin staining was detected in 48, 24 and $7 \%$ of their cases, respectively. Although these authors also reported a high frequency of cytoplasmic staining with a reciprocal reduction of membranous staining (which is also the case in our study), we believe that cytoplasmic staining only in the absence of nuclear expression does not accurately reflect the status of either adenomatous polyposis coli or $\beta$-catenin mutations. This is so because the oncogenic effect of $\beta$-catenin clearly requires its nuclear localization for its transactivating activity. ${ }^{24,25}$ The remarkably high degree of agreement between the frequency of $\beta$-catenin nuclear expression in colorectal adenocarcinomas demonstrated in our study and the documented mutation rate in the adenomatous polyposis coli and $\beta$-catenin genes in the literature further supports this notion.

The reported frequency of DNA mismatch repair gene mutations in small intestinal adenocarcinoma has been consistently low, ranging from 5 to $45 \%$ by polymerase chain reaction, ${ }^{15,18,26-29}$ and 0 to $26 \%$ by immunohistochemistry. ${ }^{18,23,27,29}$ Our immunohistochemical results are comparable to these findings. In addition, our data demonstrate a mutual exclusion between aberrant adenomatous polyposis coli and $\beta$-catenin expression and loss of immunoreactivity for mismatch repair enzymes in both small intestinal and colorectal adenocarcinomas, save for two colorectal tumors where loss of stains for both adenomatous polyposis coli and hMLH1 proteins was concurrent. This observation suggests that the Wnt and microsatellite instability pathways are indepen- dently deregulated during small intestinal tumorigenesis, analogous to that seen in colorectal adenocarcinoma.

Both p53 and RB are gatekeeper tumor suppressors that are frequently mutated in human cancers. ${ }^{30}$ Similar to that occurring in the adenoma-carcinoma sequence of colorectal tumorigenesis, ${ }^{31,32}$ p53 mutation has been proposed as a late event during the development of small intestinal adenocarcinoma, which may serve a role in tumor progression. ${ }^{15,33}$ The reported frequency of p53 mutation in small intestinal adenocarcinoma ranges from 0 to $43 \%$ by DNA-based analysis, ${ }^{15,16,34,35}$ and 24 to $65 \%$ by immunohistochemistry. ${ }^{15,18,23,33,36}$ Our data are in line with these observations and further demonstrate that in both small intestinal and colorectal adenocarcinomas, p53 overexpression, an indirect indication of p53 gene mutation, occurs only rarely in microsatellite instable tumors. In addition, our study shows that loss of RB protein expression, presumably secondary to mutations or deletion of its gene, is a rare event in small intestinal and colorectal adenocarcinomas, suggesting that $R B$ mutation does not serve a critical role in intestinal tumorigenesis.

In summary, the data presented in this report extend our previous observations ${ }^{4,5}$ that small intestinal adenocarcinoma differs markedly from colorectal adenocarcinoma tumorigenetically. By systematically examining different tumorigenic pathways well-established for colorectal tumorigenesis, we demonstrate that deregulation of the Wnt signaling pathway does not appear to be a major defect in small intestinal adenocarcinoma. Over $90 \%$ colorectal adenocarcinomas included in our study involve deregulation of the Wnt and microsatellite instability pathways, but these defects are seen in only $40 \%$ small intestinal adenocarcinomas $(P<0.0001)$. Therefore, small intestinal tumorigenesis may follow a distinct, yet unidentified, molecular pathway(s) from its colorectal counterpart despite their morphologic similarity.

\section{Acknowledgement}

We thank Ms Prosperidad Amargo for her excellent technical assistance.

\section{References}

1 Delaunoit T, Neczyporenko F, Limburg PJ, et al. Pathogenesis and risk factors of small bowel adenocarcinoma: a colorectal cancer sibling? Am J Gastroenterol 2005;100:703-710.

2 O’Riordan BG, Vilor M, Herrera L. Small bowel tumors: an overview. Dig Dis 1996;14:245-257.

3 Gill SS, Heuman DM, Mihas AA. Small intestinal neoplasms. J Clin Gastroenterol 2001;33:267-282. 
4 Chen ZM, Wang HL. Alteration of cytokeratin 7 and cytokeratin 20 expression profile is uniquely associated with tumorigenesis of primary adenocarcinoma of the small intestine. Am J Surg Pathol 2004;28:1352-1359.

5 Chen ZM, Ritter JH, Wang HL. Differential expression of $\alpha$-methylacyl coenzyme A racemase in adenocarcinomas of the small and large intestines. Am J Surg Pathol 2005;29:890-896.

6 Kolligs FT, Bommer G, Göke B. Wnt/beta-catenin/tcf signaling: a critical pathway in gastrointestinal tumorigenesis. Digestion 2002;66:131-144.

7 Giles RH, van Es JH, Clevers H. Caught up in a Wnt storm: Wnt signaling in cancer. Biochim Biophys Acta 2003;1653:1-24.

8 Behrens J, Lustig B. The Wnt connection to tumorigenesis. Int J Dev Biol 2004;48:477-487.

9 Sparks AB, Morin PJ, Vogelstein B, et al. Mutational analysis of the APC/ $\beta$-catenin/Tcf pathway in colorectal cancer. Cancer Res 1998;58:1130-1134.

10 Liu B, Nicolaides NC, Markowitz S, et al. Mismatch repair gene defects in sporadic colorectal cancers with microsatellite instability. Nat Genet 1995;9:48-55.

11 Popat S, Hubner R, Houlston RS. Systematic review of microsatellite instability and colorectal cancer prognosis. J Clin Oncol 2005;23:609-618.

12 Gologan A, Sepulveda AR. Microsatellite instability and DNA mismatch repair deficiency testing in hereditary and sporadic gastrointestinal cancers. Clin Lab Med 2005;25:179-196.

13 Wang HL, Wang J, Xiao SY, et al. Elevated protein expression of cyclin D1 and Fra-1 but decreased expression of c-Myc in human colorectal adenocarcinomas overexpressing $\beta$-catenin. Int J Cancer 2002;101: 301-310.

14 Hassan A, Yerian LM, Kuan SF, et al. Immunohistochemical evaluation of adenomatous polyposis coli, $\beta$-catenin, c-Myc, cyclin D1, p53, and retinoblastoma protein expression in syndromic and sporadic fundic gland polyps. Hum Pathol 2004;35:328-334.

15 Rashid A, Hamilton SR. Genetic alterations in sporadic and Crohn's-associated adenocarcinomas of the small intestine. Gastroenterology 1997;113:127-135.

16 Arai M, Shimizu S, Imai Y, et al. Mutations of the Kiras, p53 and $A P C$ genes in adenocarcinomas of the human small intestine. Int J Cancer 1997;70:390-395.

17 Bläker H, Helmchen B, Bönisch A, et al. Mutational activation of the RAS-RAF-MAPK and the Wnt pathway in small intestinal adenocarcinomas. Scand J Gastroenterol 2004;39:748-753.

18 Wheeler JM, Warren BF, Mortensen NJ, et al. An insight into the genetic pathway of adenocarcinoma of the small intestine. Gut 2002;50:218-223.

19 Laurent-Puig P, Beroud C, Soussi T. APC gene: database of germline and somatic mutations in human tumors and cell lines. Nucleic Acids Res 1998;26:269-270.
20 Nagase H, Nakamura Y. Mutations of the APC (adenomatous polyposis coli) gene. Hum Mutat 1993;2:425-434.

21 Powell SM, Petersen GM, Krush AJ, et al. Molecular diagnosis of familial adenomatous polyposis. N Engl J Med 1993;329:1982-1987.

22 Kinzler KW, Vogelstein B. Lessons from hereditary colorectal cancer. Cell 1996;87:159-170.

23 Svrcek M, Jourdon F, Sebbagh N, et al. Immunohistochemical analysis of adenocarcinoma of the small intestine: a tissue microarray study. J Clin Pathol 2003;56:898-903.

24 Fuchs SY, Ougolkov AV, Spiegelman VS, et al. Oncogenic $\beta$-catenin signaling networks in colorectal cancer. Cell Cycle 2005;4:1522-1539.

25 Gregorieff A, Clevers H. Wnt signaling in the intestinal epithelium: from endoderm to cancer. Genes Dev 2005;19:877-890.

26 Hibi K, Kondo K, Akiyama S, et al. Frequent genetic instability in small intestinal carcinomas. Jpn J Cancer Res 1995;86:357-360.

27 Planck M, Ericson K, Piotrowska Z, et al. Microsatellite instability and expression of MLH1 and MSH2 in carcinomas of the small intestine. Cancer 2003;97: 1551-1557.

28 Bläker H, von Herbay A, Penzel R, et al. Genetics of adenocarcinomas of the small intestine: frequent deletions at chromosome $18 \mathrm{q}$ and mutations of the SMAD4 gene. Oncogene 2002;21:158-164.

29 Brueckl WM, Heinze E, Milsmann C, et al. Prognostic significance of microsatellite instability in curatively resected adenocarcinoma of the small intestine. Cancer Lett 2004;203:181-190.

30 Hahn WC, Weinberg RA. Rules for making human tumor cells. N Engl J Med 2002;347:1593-1603.

31 Blondal JA, Benchimol S. The role of p53 in tumor progression. Semin Cancer Biol 1994;5:177-186.

32 Iacopetta B. TP53 mutation in colorectal cancer. Hum Mutat 2003;21:271-276.

33 Arber N, Hibshoosh H, Yasui W, et al. Abnormalities in the expression of cell cycle-related proteins in tumors of the small bowel. Cancer Epidemiol Biomarkers Prev 1999;8:1101-1105.

34 Mitomi H, Nakamura T, Ihara A, et al. Frequent Ki-ras mutations and transforming growth factor- $\alpha$ expression in adenocarcinomas of the small intestine: report of 7 cases. Dig Dis Sci 2003;48:203-209.

35 Norum Pedersen L, Kaerlev L, Stubbe Teglbjaerg P, et al. Glutathione $S$-transferase genotype and p53 mutations in adenocarcinoma of the small intestine. Scand J Gastroenterol 2003;38:845-849.

36 Nishiyama K, Yao T, Yonemasu H, et al. Overexpression of p53 protein and point mutation of K-ras genes in primary carcinoma of the small intestine. Oncol Rep 2002;9:293-300. 